\title{
Cor triatriatum dexter, a very rare congenital anomaly presented in a complex pathological context - case presentation
}

\author{
Gozar Liliana, Blesneac Cristina, Toganel Rodica \\ University of Medicine and Pharmacy Tirgu Mures
}

Background: Cor triatriatum dexter is an extremely rare congenital anomaly, and in most cases, without hemodynamic significance. Congenital cytomegalovirus infection is the most common viral infection, the majority of infected infants being asymptomatic and only $5-15 \%$ being symptomatic in the neonatal period.

Case report: We present the case of a female infant, aged 3 months. Clinical examination reveals cranial and facial anomalies. The karyotype was normal. Laboratory tests revealed the presence of anti-CMV antibodies in urine. Imaging studies showed cerebral and renal anomalies. Echocardiographic exam revealed a congenital anatomical feature without hemodynamic significance - cor triatriatum dexter.

Conclusions: although cytomegalovirus infection is mostly asymptomatic, it can present as a complex pathological association, in which a congenital cardiac anatomical feature without hemodynamic significance, considered by some authors as an anatomical variant, can appear.

Keywords: cor triatriatum, cytomegalovirus, infant.

Received: 06 July 2014 / Accepted: 10 August 2014

\section{Introduction}

Cor triatriatum dexter is an extremely rare congenital anomaly, and in most cases, without hemodynamic significance. The partitioning of right atrium by a septum that results from lack of normal regression of the embryonic right valve of the sinus venosus, gives rise to the triatrial heart.

Congenital cytomegalovirus (CMV) infection is the most common viral infection and most infected infants are asymptomatic. Approximately 5-15\% has neurologic deficits, hearing loss, neurodevelopment retardation [1].

In this paper we present the case of an infant with cor triatriatum dexter and congenital cytomegalovirus infection with important neurological impairment.

\section{Case report}

We present the case of a female infant, aged 3 months, born following a pathological pregnancy with placental abruption, delivered by caesarean section, low birth weight $(1550 \mathrm{~g})$ and difficult postpartum adjustment, Apgar score 6 at 1 minute, 8 at 5 minutes and 10 at 10 minutes, with respiratory distress, which required ventilation with positive pressure by nasal CPAP for 4 days, antibiotics, parenteral nourishment.

Postnatal clinical examination reveals microcephaly (cranial perimeter $31 \mathrm{~cm}$ ), facial dysmorphism, antimongoloid palpebral fissure, hypertelorism, micrognathia, lowset ears, wide anterior fontanelle, and superposition of cranial bones.

\section{Laboratory and imaging studies:}

Normal karyotype

Nephrologic consultation, abdominal ultrasound - reveals left renal agenesis and dysplastic right kidney, but with normal values of renal function tests.

Transfontanelar ultrasound: midline interhemispheric line. Symmetrical lateral ventricles. Biventricular diameter $30.4 \mathrm{~mm}$. Biparietal diameter of $96.7 \mathrm{~mm}$. IIIrd ventricle $14.4 \mathrm{~mm}$. IVth ventricle of normal appearance. Corpus callosum is present in sagittal section, but absent in the anterior portion. Cavum septum pellucidum cannot be visualized, mild frontal atrophy. Conclusion: hypoplasia of the anterior portion of the corpus callosum.

Cranial CT: craniocephalic asymmetry with moderate encephalomalacia, partial agenesis of the corpus callosum, moderate dilation of the lateral ventricles and of the IIIrd ventricle.

TORCH syndrome - anti-toxoplasma, anti-rubella and anti-CMV antibodies (ELISA) are negative, anti-CMV antibodies in urine - three consecutive samples - strongly positive, the mother was not tested antenatally but postnatally she was tested positive for IgG anti-CMV antibody.

Given the moderate functional respiratory syndrome, feeding problems, the IIIrd grade left parasternal systolic murmur detected in the first days of life, the suspicion of congenital heart disease increased, and the patient was referred to our service for cardiologic evaluation and determination of the therapeutic approach.

Chest roentgenography: global cardiomegaly and increased pulmonary vascular markings.

Echocardiography highlights the following features: the presence of the azygos vein draining into dilated superior 
vena cava (Figure 1); a prominent eustachian valve which apparently divides the right atrium, without hemodynamic significance (Figure 2, Figure 3); aneurysmal dilated pulmonary artery (Figure 4); persistent ductus arteriosus of $4-5 \mathrm{~mm}$ in diameter (Figure 4) and coronary fistulas: left

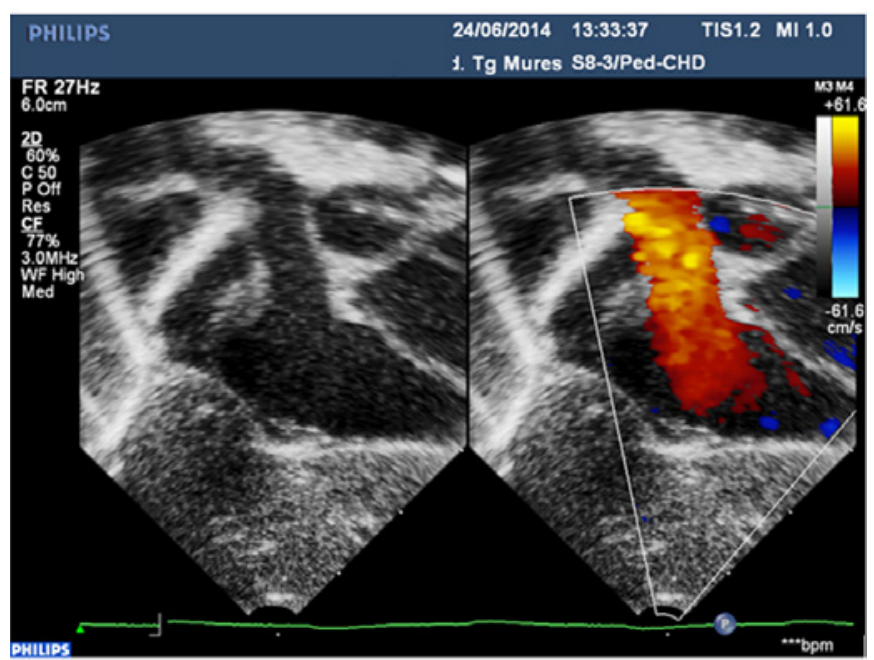

Fig. 1. Subcostal view, anatomical position - we can visualize the membrane, superior vena cava drainage into the RA, dilated superior vena cava.

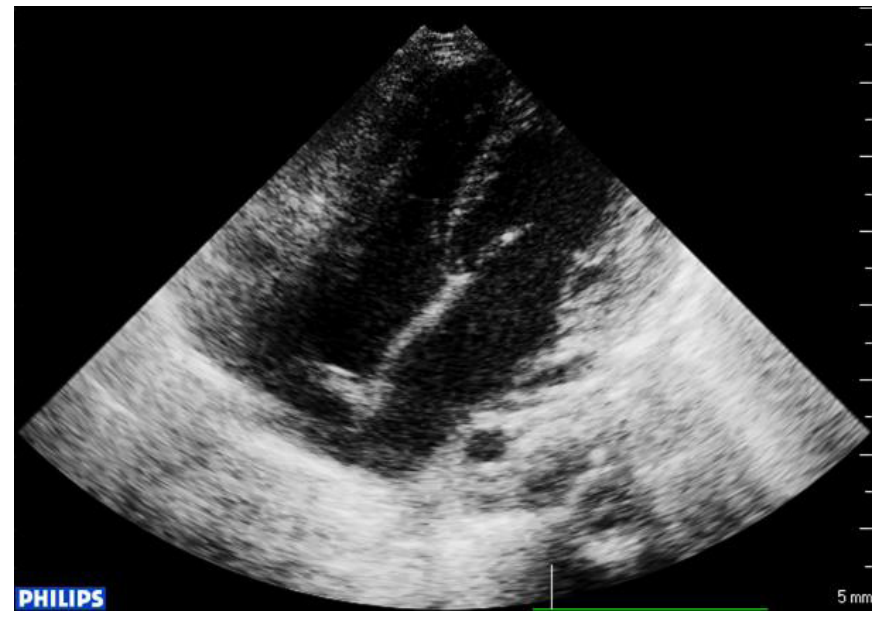

Fig. 2. Apical 4-chamber view with right atrium (RA) on the left side of the image; in the RA a membrane can be visualized.

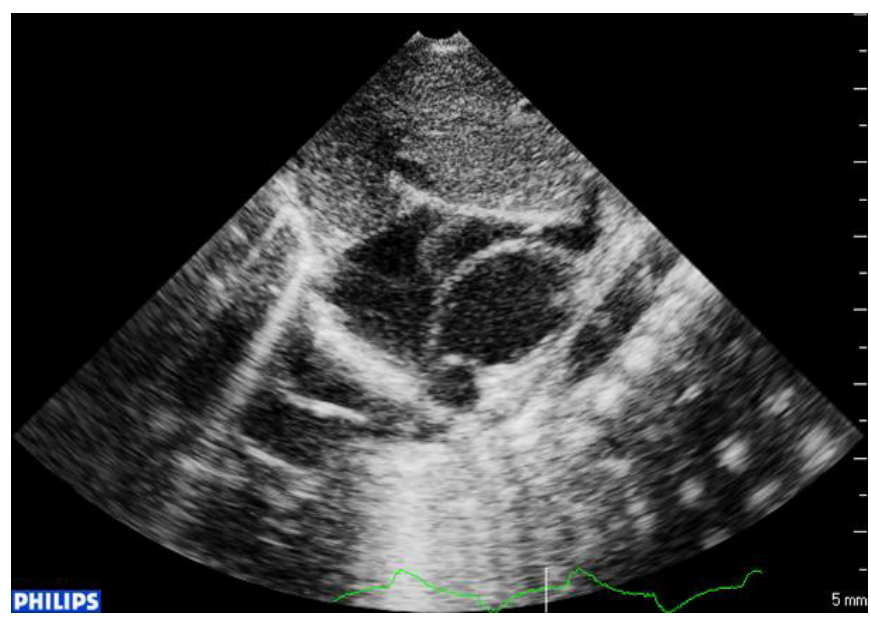

Fig. 3. Subcostal view - junction between VCl and RA and insertion of the membrane on the septum. coronary artery opening in the right ventricle, without hemodynamic significance (Figure 5).

\section{Discussions}

Congenital cytomegalovirus infection is present in approximately $1 \%$ of newborns, the majority being asymptomatic and only $5-15 \%$ being symptomatic in the neonatal period [1].

Primary infection with cytomegalovirus carries a significantly higher risk of neonatal infection compared with recurrent maternal infection [1].

In the presented case the mother was not tested before birth, but IgG anti-CMV antibodies were detected in the postnatal period.

Symptomatic infected newborns often have the following clinical manifestations: low birthweight for gestational age, cutaneous purpura and petechiae, jaundice [2]. Neurological impairment is one of the stigmata of congenital

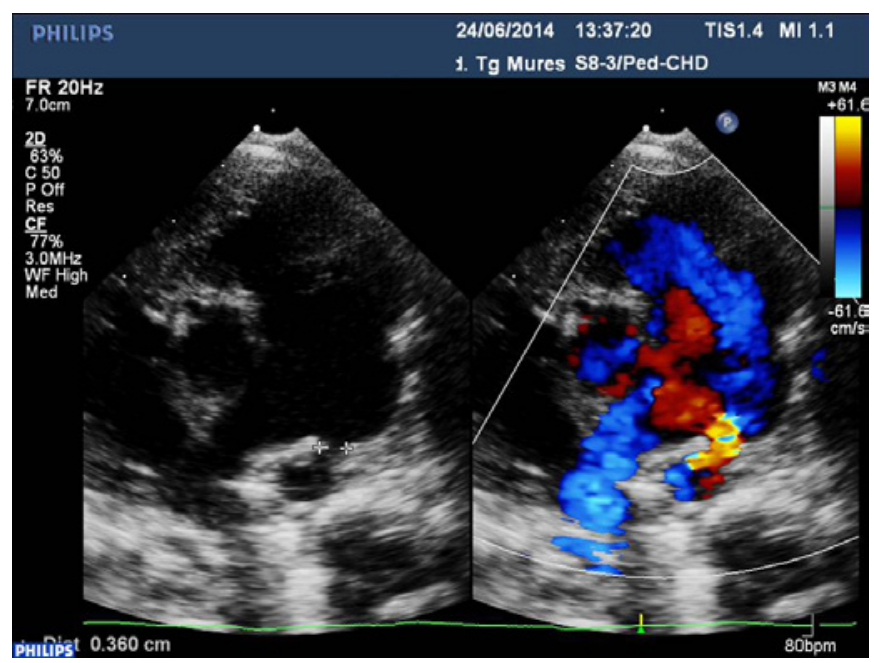

Fig. 4. Parasternal short axis view - dilated pulmonary artery and persistent ductus arteriosus. Figure 4: Parasternal short axis view - dilated pulmonary artery and persistent ductus arteriosus.

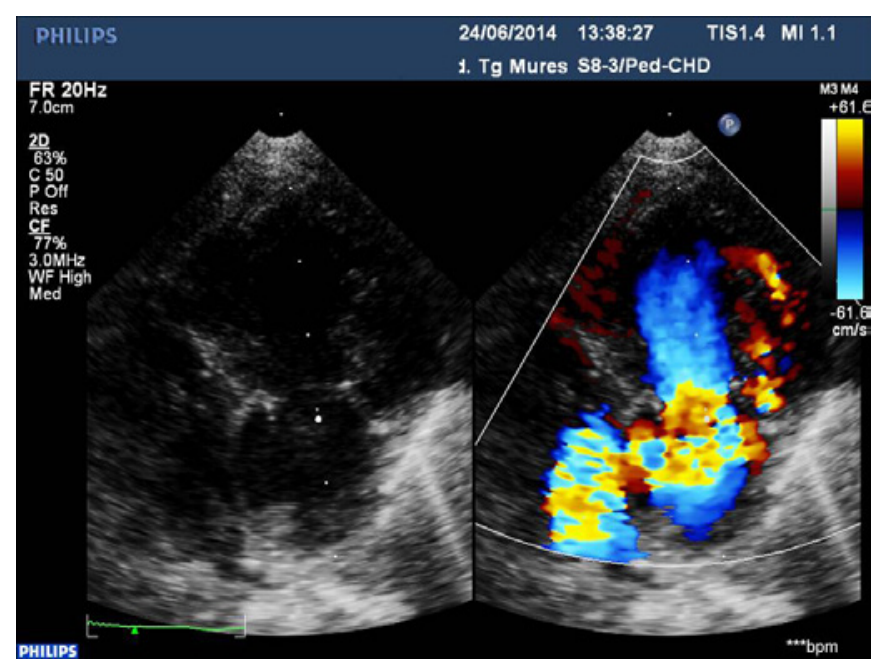

Fig. 5. Parasternal short axis view oriented toward right ventricular outflow tract - color Doppler flow showing communication between left coronary artery and right ventricle 
cytomegalovirus infection and it is manifested by microcephaly, feeding difficulties, hearing loss, seizures, neurodevelopmental retardation.

Imaging studies can show intracerebral calcifications (the most common abnormality) periventricular leukomalacia, cystic abnormalities, ventriculomegaly, hydranencephaly $[3,4]$. In the case presented here, clinical findings were microcephaly and neurodevelopmental retardation, and imaging studies showed encephalomalacia and partial agenesis of the corpus callosum. These neurological changes are rarely described in the literature as associated to congenital citomegalovirus infection.

Besides neurological impairment, symptomatic infants infected with cytomegalovirus show hematological and ocular abnormalities, endocrinopathies, abnormalities that were not present in this case. In contrast, in the presented case, congenital kidney (renal agenesis) and heart defects were present.

Cor triatriatum dexter is an extremely rare congenital anomaly [5]. In 1963, Doucette and Knoblich reported 8 cases diagnosed during necropsies [6]. Later, in 1972 Hansing and colleagues reported one case that was diagnosed and operated [6]. Studies published in the literature report less than $0.1 \%$ incidence of cor triatriatum [7]. Membrane located in the right atrium, apparently dividing the right atrium, is an anomaly that can be considered an anatomical variant. Eustachian valve is an embryological remnant of the valve of inferior vena cava and it appears as a fold, of varying sizes, with insertion at the junction of the inferior vena cava to the right atrium. This anatomical condition has no hemodynamic significance since the separation caused by the eustachian valve is incomplete [8].
The peculiarity of the presented case derives from the complex pathological context in which a congenital anatomical feature without hemodynamic significance has appeared, considered by some authors as an anatomical variant.

\section{Conclusions}

Although cytomegalovirus infection is mostly asymptomatic, it can present as a complex pathological association, in which a congenital cardiac anatomical feature without hemodynamic significance can appear.

\section{References}

1. Demmler GJ, Infectious Diseases Society of America and Centers for Disease Control. Summary of a workshop on surveillance for congenital cytomegalovirus disease, Rev Infect Dis. 1991;13(2):315-329.

2. Kylat RI, Kelly EN, Ford-Jones EL, Clinical findings and adverse outcome in neonates with symptomatic congenital cytomegalovirus (SCCMV) infection. Eur J Pediatr. 2006;165(11):773 -778.

3. Boppana SB, Fowler KB, Vaid Y, Hedlund G, Stagno S, Britt WJ, Pass RF, Neuroradiographic findings in the newborn period and long-term outcome in children with symptomatic congenital cytomegalovirus infection, Pediatrics. 1997;99(3):409 -414.

4. Noyola DE, Demmler GJ, Nelson CT, Griesser C et al., Early predictors of neurodevelopmental outcome in symptomatic congenital, cytomegalovirus infection, Houston Congenital CMV Longitudinal Study Group J Pediatr. 2001;138(3):325 -331.

5. Alboliras ET, Edwards WD, Driscoll DJ, Seward JB, Cor triatriatum dexter: Two-dimensional echocardiographic diagnosis. Journal of the American College of Cardiology, 1987, 9, 334-337.

6. Doucette J, Knoblich R, Persistent right valve of the sinus venosus, Archives of pathology, 1963; 75,105-112.

7. Arrants JE, Riopel DA, Catalano PW. Cor triatriatum: Preoperative diagnosis and successful surgical correction in a ten-week-old infant. Chest 1973;63:1027-8

8. Kim MJ, Ho Jung, anatomic variants mimicking pathology on echocardiography: differential diagnosis, J cardiovasc Ultrasound 2013; 21(3): 103-112. 